\title{
Investigation of the expression patterns and correlation of DNA methyltransferases and class I histone deacetylases in ovarian cancer tissues
}

\author{
YIFENG GU $^{1 *}$, PEIFANG YANG ${ }^{1,2 *}$, QING SHAO $^{1 *}$, XIA LIU $^{1,3}$, SHENG XIA $^{1,3}$, \\ MIAOMIAO ZHANG ${ }^{1,3}$, HUAXI XU ${ }^{1,3}$ and QIXIANG SHAO ${ }^{1,3}$ \\ ${ }^{1}$ Department of Immunology, School of Medical Science and Laboratory Medicine, Jiangsu University, Zhenjiang 212013; \\ ${ }^{2}$ Department of Gynecology and Obstetrics, Affiliated Hospital of Jiangsu University, Zhenjiang 212001; \\ ${ }^{3}$ Zhenjiang Key Laboratory of Medical Diagnosis, Zhenjiang 212001, Jiangsu, P.R. China
}

Received August 25, 2012; Accepted October 29, 2012

DOI: $10.3892 / \mathrm{ol} .2012 .1057$

\begin{abstract}
Recent studies have reported that DNA methyltransferases (DNMTs) and histone deacetylases (HDACs) are involved in the epigenetic regulation of cancer, as well as promoting cell proliferation and tumorigenesis. These mechanisms also play important roles in ovarian cancer, but little is known concerning the correlation of DNMTs and HDACs in ovarian cancer. In the present study, we used quantitative real-time reverse transcription polymerase chain reaction (qRT-PCR) and immunohistochemical staining to examine the mRNA and protein expression of DNMTs and class I HDACs of tissues from 22 cases of ovarian cancer and 8 normal ovaries as a control. Furthermore, we assessed the correlation with clinicopathological stages and the mRNA expression of these genes. The results indicated that the mRNA expression of DNMT1, DNMT3b and class I HDACs was increased in ovarian cancers, while the expression of DNMT3a was not different between cancer tissues and normal ovaries. Additionally, the results of immunohistochemical staining demonstrated that DNMT1 and DNMT3b were significantly increased in ovarian cancer samples. Furthermore, the expression of DNMT1, DNMT3b, HDAC1 and HDAC2 was significantly higher in stage III/IV compared with stage I/II ovarian carcinomas. The expression of HDAC2 was positively correlated with HDCA1, HDAC3 and HDAC8, and DNMT1
\end{abstract}

Correspondence to: Dr Sheng Xia or Professor Qixiang Shao, Department of Immunology, School of Medical Science and Laboratory Medicine, Jiangsu University, 301 Xuefu Road, Zhenjiang 212013, Jiangsu, P.R. China

E-mail: xiasheng01@yahoo.com.cn

E-mail: shao_qx@ujs.edu.cn

*Contributed equally

Key words: ovarian cancer, DNA methyltransferases, histone deacetylases, expression pattern, correlation was positively correlated with DNMT3b. Simultaneously, DNMT3b was correlated with HDAC1 and HDAC2. HDAC1 may upregulate the expression of DNMTs, but this requires confirmation by in vitro and in vivo experiments. The overall high rate of expression for class I HDACs, DNMT1 and DNMT3b suggested that these mRNAs should be explored as predictive factors in ovarian cancer. In addition, HDAC1, HDAC2 and DNMT3b cooperated in controlling ovarian cancer progression. Determining the correlations between HDACs and DNMTs in ovarian cancer will not only further clarify the mechanisms of genesis and development, but also guide clinical therapy using the inhibitors of HDACs and DNMTs.

\section{Introduction}

Ovarian cancer is the most lethal gynecological malignancy and the fifth leading cause of cancer-related mortality among females, as well as the ninth most common cancer among females. Its incidence rate ranks third, following that of cervical and uterine cancer. Due to the lack of early symptoms, $>70 \%$ of patients are diagnosed with advanced-stage disease, by which stage the cancer has spread into adjacent tissues and organs beyond the ovaries (1). Furthermore, $30-40 \%$ of patients will succumb to ovarian cancer even in the early stage. For these reasons, the mortality rate of ovarian cancer ranks first among the various tumors affecting females. The five-year survival rate of ovarian cancer is $<20 \%$, and has shown only modest improvement over the past 40 years (2). The most advanced stage ovarian cancer patients are sensitive to standard chemotherapies, but relapse occurs in over $70 \%$ of patients, resulting in chemoresistant, fatal disease (3). Therefore, a better understanding of the molecular pathogenesis of ovarian cancer will contribute to identifying novel biomarkers in the early stage, developing new therapeutic targets, and possibly increasing the five-year survival rate of ovarian cancer, thus saving the lives of many patients (4).

Epigenetic aberrations are common in the development and progression of cancer cells and are mediated by DNA methyltransferases (DNMTs), histone deacetylases (HDACs) and 
microRNA (miRNA). These modifications alter gene function and malignant cellular transformation. DNA methylation is a reversible reaction, catalyzed by three major DNMTs. One is DNMT1, which preserves the methylation patterns throughout each cell division $(5,6)$. The others are DNMT3a and DNMT3b, which transfer a methyl group to previously unmethylated genomic regions (7). Methylation occurs in specific genomic areas called $\mathrm{CpG}$ islands $(8,9)$, usually at the promoter region of a gene, and prevents gene expression.

Histone modifications, particularly acetylation and deacetylation, are the major driving force for epigenetic gene regulation. They may regulate gene transcription by regulating acetylation of DNA sequence-specific transcription factors. Examples include p53, E2F and Sp3, where deacetylation has been linked to reduced DNA binding or transcriptional activity (10-12). To date, 18 members of human HDACs have been identified and categorized into four classes, based on homology to yeast HDACs and phylogenetic analysis $(13,14)$. In general, class I HDACs (HDACs 1-3 and 8) are primarily located in the nucleus and are associated with transcriptional repressors and cofactors.

Overexpression of DNMTs and HDACs has been reported in various types of cancers including ovarian cancer (15-21). Recently, a number of studies have demonstrated that DNMTs and HDACs contribute to the epigenetic regulation in cancer cells. The results of investigations by Zhou et al (22) and You et al (23) have suggested that HDACs may have the capacity to upregulate the expression of DNMTs. Fuks et al found that HDAC1 has the ability to bind DNMT1 and purify methyltransferase activity from nuclear extracts. Moreover, DNMT1 has a transcriptional repression domain, and directly recruits histone deacetylase activity (24). Clinical trials have demonstrated that DNMT and HDAC inhibitors may be effective reagents for cancer therapy $(25,26)$. Therefore it is important to investigate the expression pattern and correlation of DNMTs and HDACs in cancer and to guide clinical anticancer therapy.

In our study, we investigated the expression levels of DNMTs and class I HDACs in ovarian cancer tissues with quantitative real-time reverse transcription polymerase chain reaction (qRT-PCR) and immunohistochemical staining, and analyzed the correlation of DNMTs and HDACs. The relevant mechanisms of DNMT and HDAC collaboration in ovarian cancer await further clarification.

\section{Materials and methods}

Antibodies and chemical reagents. Histostain ${ }^{\mathrm{TM}}$-Plus Kit (Cat. No. SP/9001) was a product of Zymed Laboratories Inc. (San Francisco, CA, USA) and purchased from Sizhengbai Biotech Company Ltd. (Beijing, China). Rabbit anti-human DNMT1 polyclonal antibody (Cat. No. ab19905) was purchased from Abcam plc. (Cambridge, UK). Rabbit anti-human DNMT3b polyclonal antibody (Q-25, Cat. No. sc-130740) was bought from Santa Cruz Biotechnology (Santa Cruz, CA, USA). TRIzol $^{\mathrm{TM}}$ reagent was purchased from Life Technologies Co. (Shanghai, China). SuperScript ${ }^{\mathrm{TM}}$ one-step RT-PCR kit was bought from Toyobo (Shanghai) Bio Co., Ltd. (Shanghai, China). SYBR-Green mix reagent was a product of Takara Bio (Dalian) Co., Ltd. (Dalian, China).
Patients and tissue samples. A total of 22 freshly resected ovarian cancer tissue samples and eight normal ovarian tissue samples (from ovariectomized patients suffering from other gynecological diseases) were collected at the Affiliated Hospital of Jiangsu University, Zhenjiang, China, in accordance with institutional guidelines and immediately frozen in liquid nitrogen for further analysis. The study was approved by Jiangsu University's ethical review committee and informed consent for the use of tissues was obtained for all individuals. The histopathological diagnosis was based on WHO criteria; the samples were assigned a grade based on Gynecologic Oncology Group criteria (27) and staged according to the International Federation of Gynecology and Obstetrics system (FIGO) (28).

The mean ages of normal and cancer patients were 54 years (range, 36 to 70 years) and 59 years (range, 37 to 76 years), respectively. Stage breakdown was: 2 (9\%) in stage I, 5 (22.7\%) in stage II, 9 (40.9\%) in stage III, and $6(27.3 \%)$ in stage IV. The tumor histotype was serous carcinoma in 19 patients $(86.4 \%)$ and mucinous carcinoma in $3(13.6 \%)$.

Total RNA extraction and $q R T-P C R$. RNA was extracted from frozen tissue samples using the TRIzol reagent (Life Technologies) according to the manufacturer's instructions. The dissolved RNA was stored at $-70^{\circ} \mathrm{C}$ before use. RNA quality was assessed with a NanoDrop1000 spectrophotometer (Eppendorf; AG, Hamburg, Germany). RT-PCR was carried out using a SuperScript ${ }^{\mathrm{TM}}$ one-step RT-PCR kit (Toyobo) according to the manufacturer's instructions. cDNA was synthesized by utilizing an oligo (dT) primer from $1 \mu \mathrm{g}$ total RNA at $42^{\circ} \mathrm{C}$ for $20 \mathrm{~min}$, followed by inactivation of the reverse transcriptase at $94^{\circ} \mathrm{C}$ for $5 \mathrm{~min}$. The qRT-PCR was performed in a final volume of $20 \mu \mathrm{l}$ containing $10 \mu \mathrm{l}$ SYBRGreen mix reagent (Takara), sense and antisense primers $(0.3 \mu \mathrm{l}, 10 \mathrm{mM})$, and cDNA $(1 \mu \mathrm{l})$ and DNA-free water $(8.4 \mu \mathrm{l})$. Primer sequences are listed in Table I. Each DNMT and HDAC value was normalized to the expression of $\beta$-actin. Values are presented as the mean \pm standard deviation (SD) of triplicate measurements.

Immunohistochemical staining. Formalin-fixed, serial paraffin-embedded ovarian cancer tissues were immunostained by the streptavidin-biotin-peroxidase complex (ABC) method, using a Histostain-Plus Kit (Zymed Laboratories Inc.). Briefly, 3- $\mu$ m-thick sections were placed on 3-aminopropyltriethoxysilane (APES)-coated slides, deparaffinized and rehydrated routinely. To unmask the antigen, sections were boiled in $0.01 \mathrm{M}$ citrate buffer ( $\mathrm{pH}$ 6.0) in a microwave oven for $20 \mathrm{~min}$. For quenching of endogenous peroxidase activity, sections were treated with $0.3 \%$ hydrogen peroxide for $10 \mathrm{~min}$ at room temperature. After rinsing the sections in phosphate-buffered saline (PBS, $\mathrm{pH} 7.4$ ), the nonspecific binding site was blocked with $10 \%$ normal goat serum for $20 \mathrm{~min}$ at room temperature. The blocking serum was discarded and then the primary antibodies were added directly. Rabbit anti-human DNMT1 polyclonal antibody (Abcam) was diluted to 1:100, rabbit anti-human DNMT3b polyclonal antibody (Santa Cruz Biotechnology) 1:50 in PBS, respectively. The sections were incubated at $4^{\circ} \mathrm{C}$ overnight. After rinsing in PBS, biotinylated goat anti-rabbit immuno- 
Table I. Characteristics of primers used for qRT-PCR.

\begin{tabular}{|c|c|c|c|}
\hline Primer & Sequence $\left(5^{\prime}-3^{\prime}\right)$ & Cycles & Annealing temp $\left({ }^{\circ} \mathrm{C}\right)$ \\
\hline \multirow[t]{2}{*}{$\beta$-actin (genebank no. NM-001101) } & Sense: CACGAAACTACCTTCAACTCC & & \\
\hline & Antisense: CATACTCCTGCTTGCTGATC & 25 & 57 \\
\hline \multirow[t]{2}{*}{ HDAC1 (genebank no. NM-004964) } & Sense: AACCTGCCTATGCTGATGCT & & \\
\hline & Antisense: CAGGCAATTCGTTTATCAGA & 30 & 57 \\
\hline \multirow[t]{2}{*}{ HDAC2 (genebank no. NM-001527) } & Sense: GGGAATACTTTCCTGGCACA & & \\
\hline & Antisense: ACGGATTGTGTAGCCACCTC & 35 & 60 \\
\hline \multirow[t]{2}{*}{ HDAC3 (genebank no. NM-003883) } & Sense: TGGCTTCTGCTATGTCAACG & & \\
\hline & Antisense: GCACGTGGGTTGGTAGAAGT & 35 & 60 \\
\hline \multirow[t]{2}{*}{ HDAC8 (genebank no. NM-001166418) } & Sense: TTTTCCCAGGAACAGGTGA & & \\
\hline & Antisense: AGCTCCCAGCTGTAAGACC & 35 & 57 \\
\hline \multirow[t]{2}{*}{ DNMT1 (genebank no. NM-001130823) } & Sense: CTACCAGGAGAAGGACAGG & & \\
\hline & Antisense: CTCACAGACGCCACATCG & 30 & 62.5 \\
\hline \multirow[t]{2}{*}{ DNMT3a (genebank no. NM-153759) } & Sense: TATGAACAGGCCGTTGGCATC & & \\
\hline & Antisense: AAGAGGTGGCGGATGACTGG & 35 & 63.5 \\
\hline \multirow[t]{2}{*}{ DNMT3b (genebank no. NM-001207056) } & Sense: TTGGGCATAAAGGTAGGAA & & \\
\hline & Antisense: CATACTCCTGCTTGCTGATC & 35 & 57 \\
\hline
\end{tabular}

$\beta$-actin gene was used as an internal standard to normalize the amount of total RNA present in each reaction. qRT-PCR, quantitative reverse transcription polymerase chain reaction; HDAC, histone deacetylase; DNMT, DNA methyltransferase.

globulin $\mathrm{G}$ (IgG) (Zymed Laboratories Inc.) was added, and the sections were incubated for $30 \mathrm{~min}$ at room temperature. After washing in PBS, peroxidase conjugated streptavidin was added and incubated for $30 \mathrm{~min}$ at room temperature. While rinsing in PBS, the peroxidase reaction was performed using $0.02 \%$ 3, 3'-diaminobenzidine tetrahydrochloride (DAB) containing PBS and $0.15 \%$ hydrogen peroxidase for 3-15 min at room temperature. Finally, tissues were stained with Mayer's hematoxylin, then dehydrated and mounted. Staining without primary antibody was used as a negative control.

Statistical analysis. The data are expressed as the mean \pm SD. Statistical analysis was performed with the Mann-Whitney $\mathrm{U}$ test between the normal group and tumor group, and we also used this statistical method in comparison of stage III/IV and stage I/II cases. Spearman's rank correlation test was used to study correlations between different genes.

\section{Results}

mRNA expression of class I HDACs, DNMT1 and DNMT3b is upregulated in ovarian cancers. The expression levels of the class I HDACs (HDAC 1, 2, 3 and 8), DNMT1, DNMT3a and DNMT3b transcripts were determined by qRT-PCR analysis and shown in Fig. 1. The relative expressions of HDAC 1, 2, 3 and 8 mRNA in ovarian cancers $(n=22)$ were significantly higher than those in normal tissues $(n=8)$, particularly for HDAC2 ( $<<0.01$; Fig. 1A). Similarly, the relative expressions of DNMT1 and DNMT3b mRNA in ovarian cancers $(n=22)$ were significantly higher than those in normal tissues $(n=8)$, particularly for DNMT1 ( $\mathrm{P}<0.01$; Fig. 1B). The relative expression of DNMT3a was not different between the two groups ( $\mathrm{P}=0.03227$; Fig. 1B middle panel).
Gene expression levels of DNMTs and HDACs were correlated with the FIGO stage. In accordance with FIGO stage, we divided the patients into two groups; stage I/II $(n=7)$ and stage III/IV $(n=15)$; and evaluated the mRNA levels at the different stages. Results showed that DNMT1 and DNMT3b expression was significantly increased in stage III/IV compared with stage I/II, particularly for DNMT1 $(\mathrm{P}<0.01$; Fig. 2A). The relative expression of HDAC1 and HDAC2 demonstrated a similar result (Fig. 2B). In contrast, the levels of HDAC3 and HDAC8 were not different between the two groups (Fig. 2B).

Protein expression levels of DNMT1 and DNMT3b were increased in ovarian cancers. The expression of DNMT1 and DNMT3b was assessed in situ on paraffin sections of normal ovarian tissues $(n=8)$ and malignant ovarian tumors $(n=22)$. Fig. 3 shows the representative immunohistochemistry results for DNMT1 and DNMT3b expression in tissues. The intensity of staining for DNMT1 and DNMT3b in malignant ovarian tumors was significantly greater than that in normal tissues (Fig. 3).

Correlation among the mRNA expression of class I HDACs, DNMT1 and DNMT3b. We examined the correlation among the expression of class I HDACs, DNMT1 and DNMT3b. The expression of HDAC2 showed a significantly positive correlation with HDAC1 $(\varrho=0.4958, \mathrm{P}=0.0309)$, HDAC3 $(\mathrm{Q}=0.4719$, $\mathrm{P}=0.0413)$ and HDAC8 $(\mathrm{Q}=0.6123, \mathrm{P}=0.0027)$. Similarly, there was a positive correlation between DNMT1 and DNMT3b ( $\mathrm{Q}=0.4736, \mathrm{P}=0.026)$. Unexpectedly, there was no correlation with HDAC1, HDAC3 and HDAC8. Notably, DNMT3b had a positive correlation with HDAC1 $(\mathrm{Q}=0.5158, \mathrm{P}=0.0238)$ and HDAC2 $(\mathrm{Q}=0.4857, \mathrm{P}=0.035$; Table II). In addition, in most ovarian cancer patients the expression of the DNMTs 
A

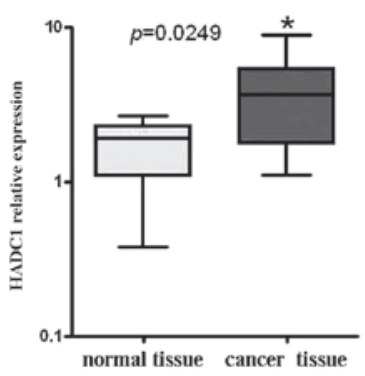

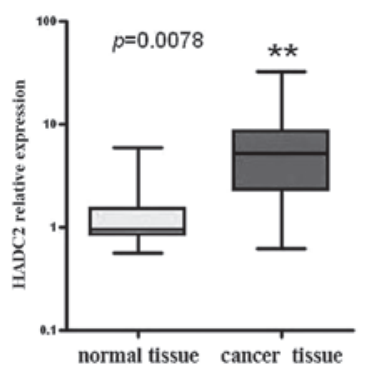

B
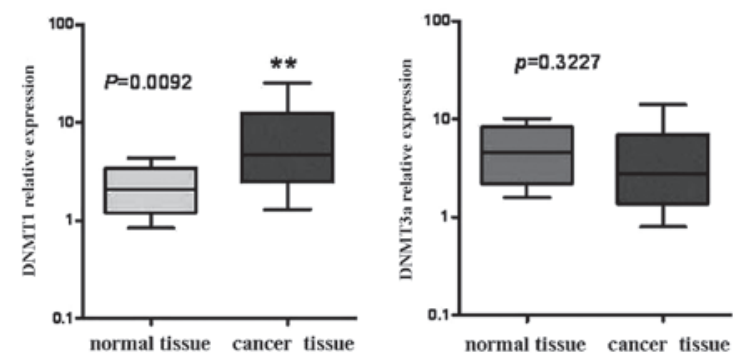
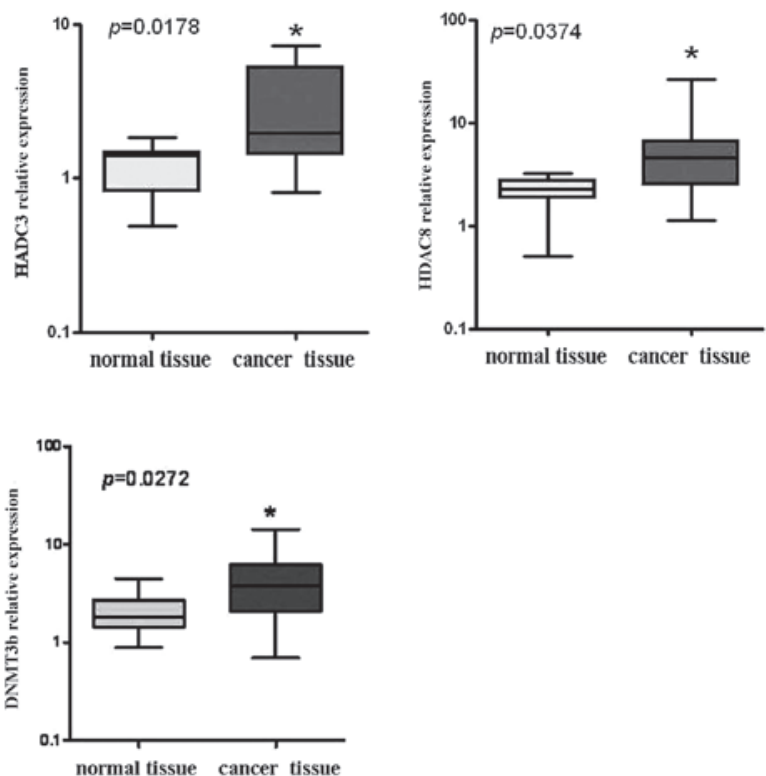

Figure 1. Relative levels of expression of (A) HDAC1, 2, 3 and 8, and (B) DNMT1, 3a and 3b mRNA by qRT-PCR in the ovarian cancer tissues ( $\mathrm{n}=22$ ), compared with the normal ovarian tissues $(n=8)$. The level of expression for HDACs and DNMTs is normalized to $\beta$-actin. For comparison of expression data between different groups the Mann-Whitney U test was used. ${ }^{*} \mathrm{P}<0.05,{ }^{* *} \mathrm{P}<0.01$. HDAC, histone deacetylase; DNMT, DNA methyltransferase; qRT-PCR, quantitative reverse transcription polymerase chain reaction.
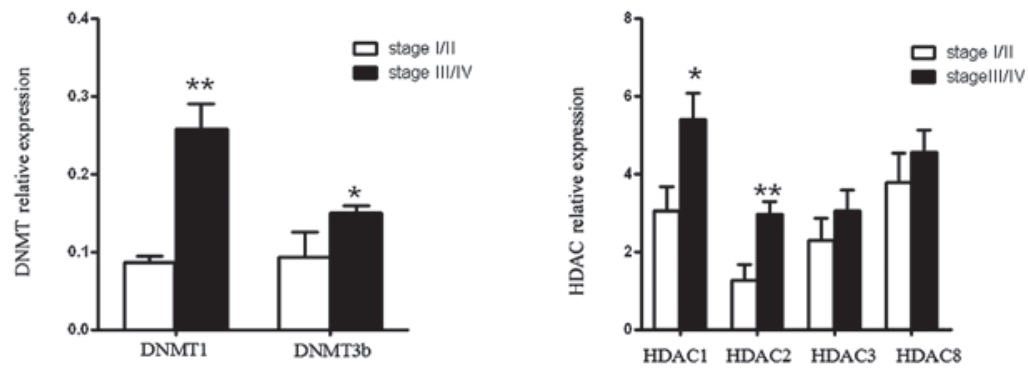

Figure 2. Positivity index for DNMT1 and $3 \mathrm{~b}$, and HDAC1, 3 and 8 in ovarian cancers according to the FIGO classification. Each value indicates the mean \pm SD. ${ }^{*} \mathrm{P}<0.05,{ }^{* *} \mathrm{P}<0.01$. DNMT, DNA methyltransferase; HDAC, histone deacetylase.

A

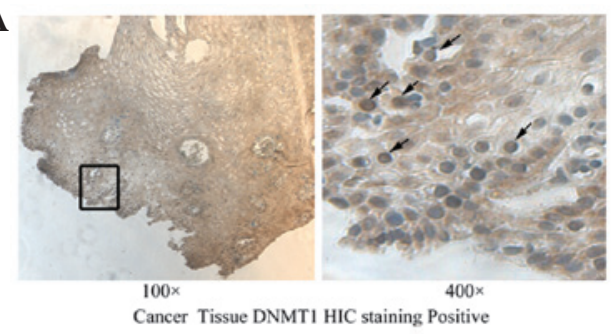

$\mathbf{C}$

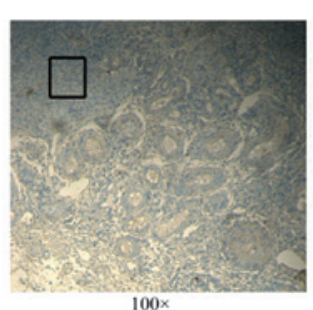

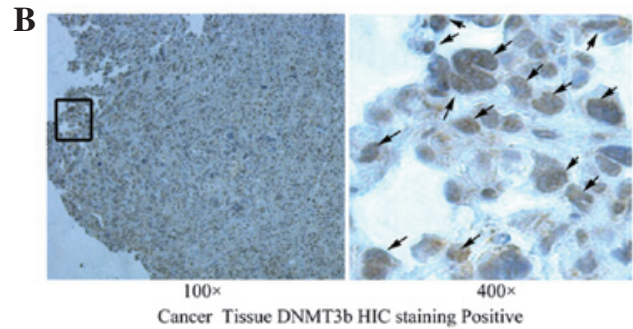

Cancer Tissue DNMT3b HIC staining Positive

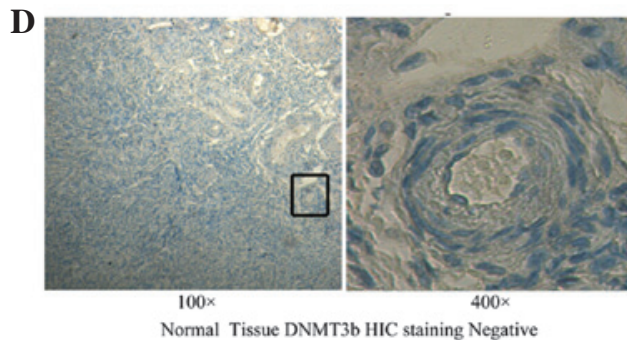

Figure 3. Immunohistochemical staining of DNMT1 and DNMT3b in ovarian tumor tissues and ovarian normal tissues. (A) Immunohistochemical staining for DNMT1 in ovarian tumor tissue, showing that some cancer cells expressed DNMT1 in nuclei (arrows indicate positive cells). (C) Immunohistochemical staining for DNMT1 in normal ovarian tissue showing no positive staining in cells. (B) Immunohistochemical staining for DNMT3b in ovarian tumor tissue showing that most of the cancer cells expressed DNMT3b in the nuclei (arrows indicate positive cells). (D) Immunohistochemical staining for DNMT3b in normal ovarian tissue showing no positive staining in cells. Magnification: Left images, x100; Right images, x400. DNMT, DNA methyltransferase. 
Table II. Spearman's correlations between the mRNA of class I HDACs, DNMT1 and DNMT3b.

\begin{tabular}{|c|c|c|c|c|c|c|c|c|}
\hline Gene & Correlation analysis & HDAC1 & HDAC2 & HDAC3 & HDAC8 & DNMT1 & DNMT3a & DNMT3b \\
\hline \multirow[t]{2}{*}{ HDAC1 } & Correlation coefficient & \multirow[t]{13}{*}{1.000} & $0.4958^{\mathrm{a}}$ & 0.3361 & -0.2027 & 0.2214 & 0.0542 & $0.5158^{\mathrm{a}}$ \\
\hline & P-value & & 0.0309 & 0.0797 & 0.4052 & 0.3622 & 0.8105 & 0.0238 \\
\hline \multirow[t]{2}{*}{ HDAC2 } & Correlation coefficient & & 1.000 & $0.4719^{\mathrm{a}}$ & $0.6123^{\mathrm{b}}$ & -0.2784 & 0.4490 & $0.4587^{\mathrm{a}}$ \\
\hline & P-value & & & 0.0413 & 0.0027 & 0.2484 & $0.0361^{\mathrm{a}}$ & 0.035 \\
\hline \multirow[t]{2}{*}{ HDAC3 } & Correlation coefficient & & & 1.000 & -0.1659 & -0.224 & 0.0616 & -0.2767 \\
\hline & P-value & & & & 0.2487 & 0.3566 & 0.7854 & 0.2515 \\
\hline \multirow[t]{2}{*}{ HDAC8 } & Correlation coefficient & & & & 1.000 & 0.2082 & 0.0311 & -0.2047 \\
\hline & P-value & & & & & 0.3924 & 0.8908 & 0.4007 \\
\hline \multirow[t]{2}{*}{ DNMT1 } & Correlation coefficient & & & & & 1.000 & 0.0734 & $0.4736^{\mathrm{a}}$ \\
\hline & P-value & & & & & & 0.7454 & 0.026 \\
\hline \multirow[t]{2}{*}{ DNMT3a } & Correlation coefficient & & & & & & 1.000 & 0.0429 \\
\hline & P-value & & & & & & & 0.8496 \\
\hline DNMT3b & $\begin{array}{c}\text { Correlation coefficient } \\
\text { P-value }\end{array}$ & & & & & & & 1.000 \\
\hline
\end{tabular}

${ }^{\mathrm{a}} \mathrm{P}<0.05,{ }^{\mathrm{b}} \mathrm{P}<0.01$. HDAC, histone deacetylase; DNMT, DNA methyltransferase.

Table III. Relative expression of class I HDACs, DNMT1 and DNMT3b in 22 samples of ovarian cancer.

\begin{tabular}{|c|c|c|c|c|c|c|c|c|}
\hline \multirow[b]{2}{*}{ Sample } & \multirow[b]{2}{*}{ Stage } & \multicolumn{7}{|c|}{ The $\mathrm{Ct}$ values for ovarian cancer tissues/the $\mathrm{Ct}$ values for normal control tissues } \\
\hline & & DNMT 1 & DNMT 3a & DNMT 3b & HDAC1 & HDAC2 & HDAC3 & HDAC8 \\
\hline 1 & I & 0.75 & 0.69 & $1.59^{\mathrm{a}}$ & $1.61^{\mathrm{b}}$ & 0.37 & 0.77 & 1.42 \\
\hline 2 & I & 1.27 & 1.18 & $1.60^{\mathrm{a}}$ & 1.27 & 1.22 & $1.95^{\mathrm{b}}$ & $2.09^{b}$ \\
\hline 3 & II & 0.62 & 0.87 & 1.02 & $1.57^{\mathrm{b}}$ & 0.55 & $2.82^{b}$ & $1.91^{\mathrm{b}}$ \\
\hline 4 & II & 0.89 & 0.73 & 0.89 & 0.64 & $1.80^{\mathrm{b}}$ & 0.65 & $2.03^{\mathrm{b}}$ \\
\hline 5 & II & $1.72^{\mathrm{a}}$ & 0.71 & 1.38 & 0.67 & 1.36 & 1.36 & 0.75 \\
\hline 6 & II & $1.61^{\mathrm{a}}$ & 1.13 & $1.87^{\mathrm{a}}$ & $2.34^{\mathrm{b}}$ & $2.04^{b}$ & $4.32^{\mathrm{d}}$ & $8.02^{\mathrm{f}}$ \\
\hline 7 & II & 1.29 & 1.42 & 1.42 & 0.89 & 0.90 & $1.58^{b}$ & 1.12 \\
\hline 8 & III & 1.43 & 0.98 & 0.93 & 1.80 & 1.60 & 0.77 & 1.36 \\
\hline 9 & III & $1.63^{\mathrm{a}}$ & 0.63 & $4.64^{\mathrm{c}}$ & $4.99^{d}$ & 0.82 & 0.82 & $3.12^{\mathrm{d}}$ \\
\hline 10 & III & $1.81^{\mathrm{a}}$ & 0.70 & 1.34 & 0.97 & 1.02 & $1.59^{\mathrm{b}}$ & $2.78^{b}$ \\
\hline 11 & III & $3.37^{\mathrm{c}}$ & 0.70 & $3.58^{\mathrm{c}}$ & $5.33^{\mathrm{d}}$ & $2.05^{\mathrm{b}}$ & 1.14 & $2.41^{b}$ \\
\hline 12 & III & $14.6^{\mathrm{e}}$ & 1.22 & $8.62^{\mathrm{e}}$ & $5.05^{\mathrm{d}}$ & $8.57^{\mathrm{f}}$ & $2.28^{\mathrm{b}}$ & $1.74^{\mathrm{b}}$ \\
\hline 13 & III & $3.10^{\mathrm{c}}$ & 0.68 & $1.72^{\mathrm{a}}$ & $2.78^{b}$ & $2.55^{\mathrm{b}}$ & $4.76^{d}$ & $3.08^{\mathrm{d}}$ \\
\hline 14 & III & 1.01 & 0.81 & $5.14^{\mathrm{c}}$ & 0.94 & $1.69^{\mathrm{b}}$ & $3.26^{\mathrm{d}}$ & 0.73 \\
\hline 15 & III & $1.68^{\mathrm{a}}$ & 1.27 & $18.12^{\mathrm{e}}$ & $3.65^{\mathrm{d}}$ & $13.21^{\mathrm{f}}$ & $5.60^{\mathrm{d}}$ & $3.11^{\mathrm{d}}$ \\
\hline 16 & III & $3.58^{c}$ & 1.34 & 1.32 & $2.50^{\mathrm{b}}$ & $2.10^{\mathrm{b}}$ & 1.46 & $1.81^{\mathrm{b}}$ \\
\hline 17 & III & $2.24^{\mathrm{a}}$ & 1.14 & $3.68^{c}$ & 1.40 & $3.70^{\mathrm{d}}$ & $2.03^{\mathrm{b}}$ & $2.17^{b}$ \\
\hline 18 & IV & $3.11^{\mathrm{c}}$ & $2.16^{\mathrm{a}}$ & $3.64^{\mathrm{c}}$ & $3.05^{\mathrm{d}}$ & $5.85^{\mathrm{d}}$ & $1.72^{\mathrm{b}}$ & $2.49^{\mathrm{b}}$ \\
\hline 19 & IV & $1.70^{\mathrm{a}}$ & $2.18^{a}$ & $1.68^{\mathrm{a}}$ & $1.84^{\mathrm{b}}$ & $5.48^{\mathrm{d}}$ & 0.65 & $10.8^{f}$ \\
\hline 20 & IV & $1.55^{\mathrm{a}}$ & 0.76 & 0.95 & 0.64 & $1.55^{\mathrm{b}}$ & 1.06 & $2.45^{\mathrm{b}}$ \\
\hline 21 & IV & $4.61^{c}$ & 0.57 & $4.40^{c}$ & $2.06^{\mathrm{b}}$ & $2.60^{\mathrm{b}}$ & $4.26^{\mathrm{d}}$ & $2.68^{b}$ \\
\hline 22 & IV & $2.50^{\mathrm{a}}$ & 1.32 & $3.12^{\mathrm{c}}$ & $1.94^{\mathrm{b}}$ & $3.40^{\mathrm{d}}$ & 0.55 & $4.60^{\mathrm{d}}$ \\
\hline
\end{tabular}

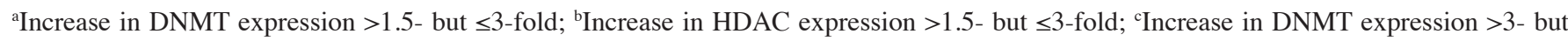
$\leq 6$-fold; 'Increase in HDAC expression $>3$ - but $\leq 6$-fold; Increase in DNMTs expression >6-fold; ' Increase in HDACs expression >6-fold. HDAC, histone deacetylase; DNMT, DNA methyltransferase.

were found to be upregulated, the expressions of HDACs are also upregulated; while the expressions of the HDACs are increased, but the expression of DNMTs are not as increased as expected (Table III). 


\section{Discussion}

Previous studies have confirmed the epigenetic regulation of the development and differentiation of the body. Epigenetic abnormalities often have a close correlation with the occurrence of a number of diseases, including cancer, alcoholic liver diseases, degenerative diseases of the nervous system, mental diseases, autoimmune diseases and cardiovascular diseases (29-31). It has been found that most cancers have abnormal DNA methylation, DNA deacetylation and miRNA expression, while the expression of DNMTs and HDACs is generally increased $(32,33)$. In mammals, DNA methylation and deacetylation are mostly mediated by DNMT1, DNMT3a, DNMT3b and class I HDAC regulation. It has been indicated that HDACs and DNMTs have important regulatory roles in human breast and cervix cancer cells. Furthermore, the inhibition of HDACs downregulates the expression of DNMT1 $(22,23)$. Ovarian cancer is one of the common malignant tumors in female reproductive organs and is associated with high expression of DNMTs and of HDACs $(4,34,35)$, but there are no studies concerning the correlation between DNMTs and HDACs in ovarian cancer.

In this study, we describe a high-level expression of class I HDAC isoforms and two functional DNMTs in ovarian cancer which catalyze cytosine methylation, and may therefore be of importance in dysregulating gene expression, in particular that of tumor suppressor genes. We used qRT-PCR assays to study the mRNA expression of DNMTs and class I HDACs in a series of 22 ovarian cancers. Overexpression of HDACs results in repression of important growth suppressive genes in numerous cancer cells, and is an important mechanism to promote cancer cell proliferation (36). Weichert et al revealed that a high proportion of ovarian carcinomas demonstrated class I HDAC protein expression using a tissue microarray, and the expression of class I HDACs indicates poor prognosis (37). High expression levels for class I HDACs were assessed by immunohistochemistry $(38,39)$, which is in line with our results. Compared with normal ovarian tissue, our results showed that HDAC2 was highly prominent $(\mathrm{P}<0.01)$. At the same time, the expression of HDAC2 was significantly higher at stage III/ IV than at stage I/II $(\mathrm{P}<0.01)$. Overexpression of HDAC2 was reportedly correlated with a more advanced stage in gastric carcinoma and it was also a prognostic indicator for a poor outcome in cases of prostate carcinoma $(16,21)$. Although these studies indicated that the increasing expression of HDAC2 was associated with tumor progression, our study is the first to demonstrate a clear correlation between HDAC 2 expression and advanced stage ovarian cancer (Table II). On the other hand, DNA hypermethylation of tumor suppressor genes in the promoter region was frequently observed in hepatocellular carcinomas (40), DNMTs are possibly responsible for DNA hypermethylation of these genes since their expression levels are known to increase during early tumorigenesis (17). We found that the mRNA and protein expression levels of DNMT1 and $3 b$ were high in ovarian cancers compared with normal tissues, while for DNMT3a there was no difference between them. The result was similar to those observed in breast cancer (41). DNMT1 was highly prominent $(\mathrm{P}<0.01)$ among these DNMTs. Furthermore, the expression of DNMT1 was significantly higher at stage III/IV than at stage I/II $(\mathrm{P}<0.01)$, supporting a role for DNMT1 in tumorigenesis (42). The mRNA expression of DNMT1, DNMT3b, HDAC1 and HDAC2 were particularly prominent in high-grade tumors in ovarian cancers, which indicated that they may be the biomarkers of tumor aggressiveness and proliferation, as their high expression is closely associated with ovarian carcinoma progression. In our results, the expression of HDAC2 was correlated with HDCA1, HDAC3 and HDAC8, and the expression of DNMT1 was correlated with DNMT3b. A notable aspect of our results is that the level of DNMT3b was correlated with HDAC1 and HDAC2 in ovarian cancer, which demonstrated that the two epigenetic events cooperated in controlling ovarian cancer progression (Table II). There have been reports that methylation of histone $\mathrm{H} 3$ lysine 9 may be triggered by DNA methylation (43), and DNMTs have also been shown to interact with HDACs, histone methyltransferases (HMTs) and methylcytosine-binding proteins in a complex network $(22,23,44)$. Our results also imply that the expression of the DNMTs are regulated by HDACs (Table III); however, these findings need to be demonstrated by further research. This finding was in accordance with the research results of Zhou et al (22) and You et al (23).

In summary, our studies demonstrate that the mRNA expression of DNMT1, DNMT3b and class I HDACs was increased in ovarian cancers. HDAC1, HDAC2, DNMT1 and DNMT3b expression increased with stage. Furthermore, HDAC1, HDAC2 and DNMT3b cooperated in controlling ovarian cancer progression and the HDACs may upregulate the expression of DNMTs. Detecting the expressions of the DNMTs and HDACs may aid the diagnosis and guide the clinical use of the inhibitors of DNMTs and HDACs to treat ovarian cancer. Further exploration is warranted to gain more information.

\section{Acknowledgements}

This study was supported by the following grants: The National Natural Science Foundation of China (Grant nos. 30671984, 8127302, 31200676 and 81172834), The Natural Science Foundation of Jiangsu Province of China (Grant no. BK2008231) and Sci-tech Innovation Team of Jiangsu University (Grant no. 2008-018-02).

\section{References}

1. Barnholtz-Sloan JS, Schwartz AG, Qureshi F, Jacques S, Malone J and Munkarah AR: Ovarian cancer: changes in patterns at diagnosis and relative survival over the last three decades. Am J Obstet Gynecol 189: 1120-1127, 2003.

2. Jemal A, Siegel R, Ward E, et al: Cancer statistics, 2008. CA Cancer J Clin 58: 71-96, 2008.

3. Liu CM: Cancer of the ovary. N Engl J Med 352: 1268-1269; author reply 1268-1269, 2005.

4. Kwon MJ and Shin YK: Epigenetic regulation of cancer-associated genes in ovarian cancer. Int J Mol Sci 12: 983-1008, 2011.

5. Li E, Bestor TH and Jaenisch R: Targeted mutation of the DNA methyltransferase gene results in embryonic lethality. Cell 69: 915-926, 1992.

6. Li E, Beard C and Jaenisch R: Role for DNA methylation in genomic imprinting. Nature 366: 362-365, 1993.

7. Okano M, Bell DW, Haber DA and Li E: DNA methyltransferases Dnmt3a and Dnmt3b are essential for de novo methylation and mammalian development. Cell 99: 247-257, 1999.

8. Weber M, Hellmann I, Stadler MB, et al: Distribution, silencing potential and evolutionary impact of promoter DNA methylation in the human genome. Nat Genet 39: 457-466, 2007. 
9. Herman JG and Baylin SB: Gene silencing in cancer in association with promoter hypermethylation. N Engl J Med 349: 2042-2054, 2003.

10. Gu W and Roeder RG: Activation of $\mathrm{p} 53$ sequence-specific DNA binding by acetylation of the p53 C-terminal domain. Cell 90 : 595-606, 1997.

11. Marzio G, Wagener C, Gutierrez MI, Cartwright P, Helin K and Giacca M: E2F family members are differentially regulated by reversible acetylation. J Biol Chem 275: 10887-10892, 2000.

12. Ammanamanchi S, Freeman JW and Brattain MG: Acetylated sp3 is a transcriptional activator. J Biol Chem 278: 35775-35780, 2003

13. de Ruijter AJ, van Gennip AH, Caron HN, Kemp S and van Kuilenburg AB: Histone deacetylases (HDACs): characterization of the classical HDAC family. Biochem J 370 (Pt 3): 737-749, 2003

14. Gregoretti IV, Lee YM and Goodson HV: Molecular evolution of the histone deacetylase family: functional implications of phylogenetic analysis. J Mol Biol 338: 17-31, 2004.

15. Mizuno S, Chijiwa T, Okamura T, et al: Expression of DNA methyltransferases DNMT1, 3A, and 3B in normal hematopoiesis and in acute and chronic myelogenous leukemia. Blood 97: 1172-1179, 2001.

16. Yang B, Guo M, Herman JG and Clark DP: Aberrant promoter methylation profiles of tumor suppressor genes in hepatocellular carcinoma. Am J Pathol 163: 1101-1107, 2003.

17. Girault I, Tozlu S, Lidereau R and Bièche I: Expression analysis of DNA methyltransferases $1,3 \mathrm{~A}$, and $3 \mathrm{~B}$ in sporadic breast carcinomas. Clin Cancer Res 9: 4415-4422, 2003.

18. Ahluwalia A, Hurteau JA, Bigsby RM and Nephew KP: DNA methylation in ovarian cancer. II. Expression of DNA methyltransferases in ovarian cancer cell lines and normal ovarian epithelial cells. Gynecol Oncol 82: 299-304, 2001.

19. Omisanjo OA, Biermann K, Hartmann S, et al: DNMT1 and HDAC1 gene expression in impaired spermatogenesis and testicular cancer. Histochem Cell Biol 127: 175-181, 2007.

20. Weichert W: HDAC expression and clinical prognosis in human malignancies. Cancer Lett 280: 168-176, 2009.

21. Weichert W, Röske A, Gekeler V, et al: Histone deacetylases 1,2 and 3 are highly expressed in prostate cancer and HDAC2 expression is associated with shorter PSA relapse time after radical prostatectomy. Br J Cancer 98: 604-610, 2008.

22. Zhou Q, Agoston AT, Atadja P, Nelson WG and Davidson NE: Inhibition of histone deacetylases promotes ubiquitin-dependent proteasomal degradation of DNA methyltransferase 1 in human breast cancer cells. Mol Cancer Res 6: 873-883, 2008.

23. You JS, Kang JK, Lee EK, et al: Histone deacetylase inhibitor apicidin downregulates DNA methyltransferase 1 expression and induces repressive histone modifications via recruitment of corepressor complex to promoter region in human cervix cancer cells. Oncogene 27: 1376-1386, 2008.

24. Fuks F, Burgers WA, Brehm A, Hughes-Davies L and Kouzarides T: DNA methyltransferase Dnmt1 associates with histone deacetylase activity. Nat Genet 24: 88-91, 2000.

25. Boumber Y and Issa JP: Epigenetics in cancer: what's the future? Oncology (Williston Park) 25: 220-226, 228, 2011.
26. Marks PA: Epigenetic targeted anti-cancer drugs: an unfolding story. Oncology (Williston Park) 25: 231, 235, 2011.

27. Benda JA and Zaino R: GOG Pathology Manual. Buffalo, NY: Gynecologic Oncology Group. 1994.

28. Staging announcement: FIGO cancer committee. Gynecol Oncol 25: 4, 1986.

29. Tsankova N, Renthal W, Kumar A and Nestler EJ: Epigenetic regulation in psychiatric disorders. Nat Rev Neurosci 8: 355-367, 2007.

30. Halusková J: Epigenetic studies in human diseases. Folia Biol (Praha) 56: 83-96, 2010

31. Mandrekar P: Epigenetic regulation in alcoholic liver disease. World J Gastroenterol 17: 2456-2464, 2011.

32. Brait M and Sidransky D: Cancer epigenetics: above and beyond. Toxicol Mech Methods 21: 275-288, 2011.

33. Ferguson LR, Tatham AL, Lin Z and Denny WA: Epigenetic regulation of gene expression as an anticancer drug target. Curr Cancer Drug Targets 11: 199-212, 2011.

34. Chen H, Hardy TM and Tollefsbol TO: Epigenomics of ovarian cancer and its chemoprevention. Front Genet 2: 67, 2011.

35. Maldonado L and Hoque MO: Epigenomics and ovarian carcinoma. Biomark Med 4: 543-570, 2010.

36. Abbas A and Gupta S: The role of histone deacetylases in prostate cancer. Epigenetics 3: 300-309, 2008.

37. Weichert W, Denkert C, Noske A, et al: Expression of class I histone deacetylases indicates poor prognosis in endometrioid subtypes of ovarian and endometrial carcinomas. Neoplasia 10: 1021-1027, 2008

38. Hayashi A, Horiuchi A, Kikuchi N, et al: Type-specific roles of histone deacetylase (HDAC) overexpression in ovarian carcinoma: HDAC1 enhances cell proliferation and HDAC3 stimulates cell migration with downregulation of E-cadherin. Int J Cancer 127: 1332-1346, 2010.

39. Song J, Noh JH, Lee JH, et al: Increased expression of histone deacetylase 2 is found in human gastric cancer. APMIS 113 264-268, 2005.

40. Sun L, Hui AM, Kanai Y, Sakamoto M and Hirohashi S: Increased DNA methyltransferase expression is associated with an early stage of human hepatocarcinogenesis. Jpn J Cancer Res 88: 1165-1170, 1997.

41. Braconi C, Huang N and Patel T: MicroRNA-dependent regulation of DNA methyltransferase-1 and tumor suppressor gene expression by interleukin-6 in human malignant cholangiocytes Hepatology 51: 881-890, 2010.

42. Wang L, Zou X, Berger AD, et al: Increased expression of histone deacetylaces (HDACs) and inhibition of prostate cancer growth and invasion by HDAC inhibitor SAHA. Am J Transl Res 1: 62-71, 2009.

43. Tariq M, Saze H, Probst AV, Lichota J, Habu Y and Paszkowski J: Erasure of $\mathrm{CpG}$ methylation in Arabidopsis alters patterns of histone H3 methylation in heterochromatin. Proc Natl Acad Sci USA 100: 8823-8827, 2003

44. Fuks F, Hurd PJ, Wolf D, Nan X, Bird AP and Kouzarides T: The methyl-CpG-binding protein MeCP2 links DNA methylation to histone methylation. J Biol Chem 278: 4035-4040, 2003. 